\title{
CONSUMO DE OXIGÊNIO ESTIMADO EM AMPUTADOS UNILATERAIS DE MEMBROS INFERIORES
}

\section{Elisabete Antunes San Martin Paloma de Borba Schneiders}

Acadêmicas do curso de fisioterapia da Universidade de Santa Cruz do Sul (UNISC), Brasil.

\section{Tatiana Lasch de Souza Ana Paula Dattein Peiter}

Fisioterapeutas pela Universidade de Santa Cruz do Sul (UNISC), Brasil.

\section{Cássia da Luz Goulart}

Doutoranda da Universidade Federal de São Carlos (UFSCAR), Brasil.

\section{Andréa Lúcia Gonçalves da Silva}

Pós-doutoranda na University of Illinois at Chicago; Universidade de Santa Cruz do Sul (UNISC), Brasil.

\section{Angela Cristina Ferreira da Silva}

Coordenadora do Departamento de Educação Física e Saúde da Universidade de Santa Cruz do Sul (UNISC), Brasil.
RESUMO: A amputação do membro inferior (MI) além de alterações anatômicas pode influenciar no gasto energético elevando o consumo de oxigênio $\left(\mathrm{VO}_{2}\right)$. Desta forma, objetivou-se estimar o $\mathrm{VO}_{2}$ de amputados unilaterais de MI e identificar as características clínicas que $o$ influenciam. Trata-se de um estudo transversal que verificou a capacidade funcional e estimou o VO2 por meio do questionário Duke Activity Status Index (DASI). Foram avaliados 28 amputados de MI

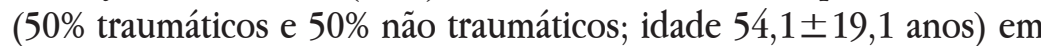
nível transfemural $(75 \%)$ e transtibial $(25 \%)$, com predominância de obesos $(42,9 \%)$ e sexo masculino $(78,6 \%)$. O escore total do DASI foi $16,7(2,7-36,7)$ pontos e o $\mathrm{VO}_{2}$ estimado $17,2 \pm 4,6 \mathrm{~mL} \cdot \mathrm{kg}^{-1} \cdot \mathrm{min}^{-1}$ caracterizando-os com capacidade funcional comprometida e $\mathrm{VO}_{2}$ muito fraco. Encontramos correlações entre $\mathrm{o} \mathrm{VO}_{2}$ estimado e a idade $(\mathrm{r}=$ $-0,516 ; p=0,005)$ da amostra total, e quando analisados por nível de amputação transfemural $(r=-0,577 ; p=0,006)$. Amputados de MI unilateral apresentaram diminuída capacidade funcional e $\mathrm{VO}_{2}$ estimado muito fraco. A idade, obesidade, nível transfemural e a causa não traumática da amputação influenciaram diretamente o baixo $\mathrm{VO}_{2}$ estimado.

PALAVRAS-CHAVE: Amputação; Consumo de oxigênio; Membro inferior; Atividade cotidiana.

\section{ESTIMATE CONSUMPTION OF OXYGEN IN PEOPLE WITH UNILATERAL AMPUTATION OF THE LOWER LIMBS}

ABSTRACT: Besides anatomical alterations, the amputation of a lower limb (LL) affects energy expenditure and increases oxygen consumption (VO2). The VO2 of LL-amputated people is estimated and the clinical characteristics affecting it are identified. Current transversal study verified the functional capacity and calculated $\mathrm{VO} 2$ by the questionnaire Duke Activity Status Index (DASI). Twenty-eight LL-amputated people were evaluated: $50 \%$ traumatic cases and 50\% non-traumatic cases; age $54.1 \pm 19.1$ years) at transfemur (75\%) and transtibia (25\%) level, predominantly obese $(42.9 \%)$ and males $(78.6 \%)$. Total DASI score was 16.7(2.7-36.7) and VO2 was estimated at 17.2 $\pm 4.6 \mathrm{~mL} . \mathrm{kg}-1 . \mathrm{min}-1$. Functional capacity was compromised and VO2 extremely weak. There was a co-relationship between estimated $\mathrm{VO} 2$ and age $(r=-0.516$; $p=0.005$ ) of total sample and when analyzed by transfemur amputation level $(r=-0.577 ; p=0.006)$. Unilateral LL-amputated people had decreased functional capacity and very weak VO2. Age, obesity, transfemur level and non-traumatic cause affected directly low estimated VO2.

KEY WORDS: Amputation; Oxygen consumption; Lower limbs; Daily activities. 


\section{INTRODUÇÃO}

A amputação está relacionada àretirada de parte do corpo com a intenção de proporcionar alívio nos sintomas, melhorar e/ou preservar funções motoras e da qualidade de vida do sujeito que necessita deste procedimento ${ }^{1}$. As principais causas de amputação são as complicações do diabetes melittus (DM), doenças vasculares, somando mais de $50 \%$ das amputações não traumáticas, sendo estas mais frequentes em homens entre 50 e 70 anos, acometendo predominantemente os membros inferiores $^{2,3}$. Em contrapartida, os acidentes de trânsito são responsáveis por cerca de $60 \%$ das causas traumáticas de amputaçãó ${ }^{4}$.

Além das alterações anatômicas, a amputação leva a redução de força e funcionalidade, mudanças de esquema corporal, disfunções de marcha, coordenação, equilíbrio e de aspectos psicossociais ${ }^{3}$ que podem influenciar no gasto energético ocasionando elevação do consumo de oxigênio $\left(\mathrm{VO}_{2}\right)$ especialmente nos amputados transfemurais de causa traumática ${ }^{5}$. Quanto mais proximal o nível da amputação maior será o gasto energético. Adicionalmente, o mesmo também depende do tipo de prótese utilizada uma vez que a evolução tecnológica protética objetiva reduzir o consumo de energia e aumentar a mobilidade dos amputados protetizados ${ }^{6}$.

Frequentemente, ${\mathrm{o} \mathrm{VO}_{2}}_{2}$ e avaliado com dispositivos sofisticados, por meio da análise de gases exalados, durante testes de capacidade de exercício ${ }^{5,7,8}$. Entretanto, as alterações biomecânicas e decorrentes da amputação ${ }^{3}$ podem dificultar a execução desta análise e o uso de mensurações indiretas e menos estressoras são de extrema importância ${ }^{9 \cdot 11}$. Neste sentido, o objetivo deste estudo foi estimar indiretamente $\mathrm{o} \mathrm{VO}_{2}$ de amputados unilaterais de membros inferiores bem como identificar as características clínicas que influenciam no $\mathrm{VO}_{2}$ destes indivíduos.

\section{METODOLOGIA}

Estudo de delineamento transversal, com amostragem de conveniência composta por 28 pacientes com amputação traumática e não traumática do Serviço de Reabilitação Física, triados na Clínica de Fisioterapia da Universidade de Santa Cruz do Sul, cidade de Santa Cruz do Sul - RS. Este estudo foi devidamente aprovada pelo Comitê de Ética em Pesquisa da Universidade de Santa Cruz do Sul com protocolo $n^{0} 1.361 .054$. Foram incluídos no estudo indivíduos adultos, de ambos os sexos, com nível de amputação unilateral transfemural e transtibial, protetizados e não protetizados. Foram excluídos os indivíduos amputados com déficit cognitivo e/ou alterações neurológicas, verificados por meio da capacidade de responder ao questionário e aqueles com amputações bilaterais ou de membros superiores.

As variáveis analisadas neste estudo foram relacionadas às características clínicas, nível de amputação e capacidade funcional percebida por meio do questionário Duke Activity Status Index (DASI) ${ }^{12,13}$. O questionário DASI tem por finalidade verificar o desempenho do sujeito para a realização de 12 itens que avaliam atividades diárias como higiene pessoal, locomoção, tarefas domésticas, função sexual e recreação, apresentando com os respectivos custos metabólicos. Cada item tem um peso específico com base no custo metabólico (MET) e sua pontuação final varia entre $0-58,2$ pontos, sendo que valores mais baixos indicam maior limitação nas atividades de vida diárias (AVDs). Portanto, quanto maior a pontuação, melhor a capacidade funcional. Posteriormente o $\mathrm{VO}_{2}$ (mL.Kg-1.min-1) é estimado por meio da seguinte equação de regressão linear múltipla: $\mathrm{VO}_{2}=0,43$ x DASI $+9,6$, de acordo com o nível de atividade física descrito pelo paciente ao responder o questionário ${ }^{12,13}$, sendo classificados conforme Cooper $(1982)^{14}$ podendo variar entre muito fraca e superior aptidão física.

Os dados foram inseridos e analisados no programa estatístico SPSS $®$ (versão 20,0). De acordo com a natureza da distribuição das variáveis utilizaram-se as medidas de tendência central e de dispersão para apresentação dos resultados: frequência, média e desvio-padrão (paramétricas) ou mediana com intervalo mínimo e máximo (não paramétricas). Foi utilizado teste T de Student para as variáveis paramétricas e Mann-Whitney para não paramétricas, as associações entre as variáveis foram avaliadas com os coeficientes de Correlação de Spearman. Para análise de proporções das variáveis categóricas utilizou-se o teste de Qui-quadrado. Foi considerado significativo um $p \leq 0,05$. 
As análises foram realizadas como amostra total e posteriormente com estratificação dos indivíduos em grupos, sendo estes por motivo de amputação em grupo traumático (GT) e grupo não traumático (GNT) e conforme o nível de amputação em grupo transtibial (GTT) e grupo transfemural (GTF) respectivamente.

\section{RESULTADOS}

As características clínicas dos amputados unilaterais de membro inferior estão descritas na Tabela 1. Observa-se que por meio do resultado do DASI os indivíduos avaliados possuem um $\mathrm{VO}_{2}$ estimado baixo, classificado como muito fraco.

Tabela 1. Características clínicas dos amputados

\begin{tabular}{|c|c|}
\hline Variáveis & Amputados $(\mathrm{n}=28)$ \\
\hline Sexo masculino, n (\%) & $22(78,6)$ \\
\hline Sexo Feminino, n (\%)f & $06(21,4)$ \\
\hline Idade (anos) & $54,1 \pm 19,1$ \\
\hline $\operatorname{IMC}\left(\mathrm{kg} / \mathrm{m}^{2}\right)^{*}$ & $29 \pm 6,1$ \\
\hline \multicolumn{2}{|l|}{ Classificação IMC I $^{\#}$ n (\%) } \\
\hline Normal & $10(35,7)$ \\
\hline Sobrepeso & $06(21,4)$ \\
\hline Obesidade & $12(42,9)$ \\
\hline Tempo de amputação (meses) & $40(18-216)$ \\
\hline \multicolumn{2}{|l|}{ Motivo da amputação, n (\%) } \\
\hline Traumático & $14(50,0)$ \\
\hline Não traumático & $14(50,0)$ \\
\hline \multicolumn{2}{|l|}{ Nível da amputação, n (\%) } \\
\hline Transtibial & $07(25,0)$ \\
\hline Transfemural & $21(75,0)$ \\
\hline \multicolumn{2}{|l|}{ Altura da amputação, n (\%) } \\
\hline Proximal & $01(3,6)$ \\
\hline Medial & $18(64,3)$ \\
\hline Distal, & $09(32,1)$ \\
\hline
\end{tabular}

\begin{tabular}{lc} 
& (Conclusão) \\
\hline Variáveis & Amputados $(\mathbf{n = 2 8 )}$ \\
\hline $\begin{array}{l}\text { Protetização, } \mathrm{n}(\%) \\
\text { Protetizado }\end{array}$ & $25(89,3)$ \\
Não protetizado & $03(10,7)$ \\
$\begin{array}{l}\text { Presença de comorbidades, } \mathrm{n} \\
(\%)\end{array}$ & $15(53,6)$ \\
$\quad$ HAS & $05(17,9)$ \\
$\quad$ Diabetes & $06(21,4)$ \\
$\quad$ HAS e diabetes & $04(14,3)$
\end{tabular}

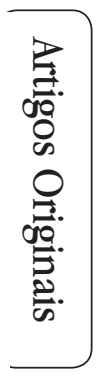

DASI

Escore total $16,7(2,7-36,7)$

$\mathrm{VO}_{2}$ estimado (mL.kg-1. $\min ^{-1}$ )

$17,2 \pm 4,6$

\section{Classificação do $\mathrm{VO}_{2,}$ n (\%)}

Muito fraca $28(100)$

Dados expressos em frequência, mediana, média \pm desvio-padrão e intervalo interquartil; \%: porcentagem; *IMC: Índice de Massa Corporal, segundo Deé, Lelovics 20125; HAS: Hipertensão Arterial Sistêmica; DASI: Duke Activity Status Index for Cardiovascular Diseases; $\mathrm{VO}_{2}$ : consumo de oxigênio; mL: mililitros; kg: quilogramas; min: minutos; ${ }^{\#}$ Classificação do $\mathrm{VO}_{2}$, segundo Cooper $1982^{14}$; Classificação do IMC, segundo WHO $2000^{16}$

No presente estudo, foram observadas cor-

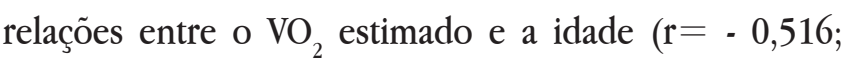
$\mathrm{p}=0,005) \mathrm{da}$ amostra como um todo, bem como entre o $\mathrm{VO}_{2}$ estimado e a idade $(\mathrm{r}=-0,577 ; \mathrm{p}=0,006)$ quando analisados por nível de amputação (Figura 1).

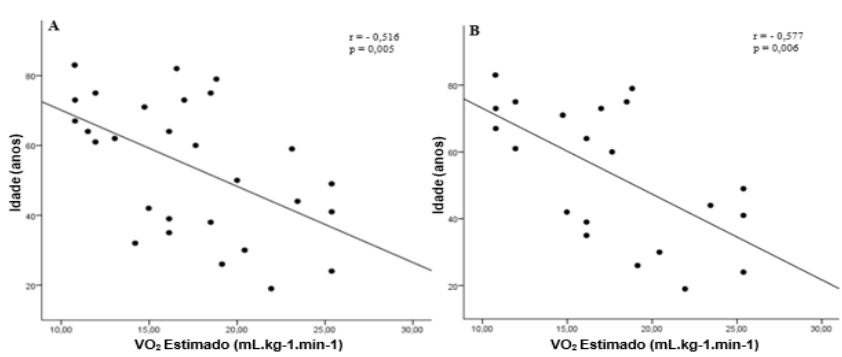

Figura 1. Relação entre o $\mathrm{VO}_{2}$ Estimado e a Idade dos amputados. A. Correlação entre o Consumo de oxigênio estimado ( $\mathrm{VO}_{2}$ estimado) e Idade (anos) dos 28 amputados avaliados. B. Correlação entre o $\mathrm{VO}_{2}$ estimado e a Idade (anos) dos amputados transfemurais $(\mathrm{n}=21)$ avaliados. Utilizou-se teste de Correlação de Spearman $(\mathrm{p}<0,05)$; mL: mililitros; kg: quilogramas; min: minutos 
Na Tabela 2 estão descritos os resultados obtidos após a estratificação dos indivíduos por motivo de amputação em grupo traumático (GT) e grupo não traumático (GNT) onde se observou que o GNT apresentou idade mais avançada e menor $\mathrm{VO}_{2}$ estimado quando comparados ao GT.

Tabela 2. Características clínicas dos amputados estratificados em GT e GNT

(Continua)

\begin{tabular}{|c|c|c|c|}
\hline \multirow[b]{2}{*}{ Variáveis } & \multicolumn{3}{|c|}{ AMPUTADOS $(\mathrm{n}=28)$} \\
\hline & $\begin{array}{c}\text { GT } \\
(n=14)\end{array}$ & $\begin{array}{c}\text { GNT } \\
(n=14)\end{array}$ & $p$ \\
\hline Sexo masculino, $\mathrm{n}(\%)^{\alpha}$ & $13(92,9)$ & $09(64,3)$ & 0,394 \\
\hline Sexo feminino, $\mathrm{n}(\%)^{\alpha}$ & $01(7,1)$ & $05(35,7)$ & 0,102 \\
\hline Idade (anos) ${ }^{\sigma}$ & $44,1 \pm 18,6$ & $64,2 \pm 14,2$ & 0,004 \\
\hline IMC $\left(\mathrm{kg} / \mathrm{m}^{2}\right)^{* \sigma}$ & $27,0 \pm 4,8$ & $30,9 \pm 6,8$ & 0,092 \\
\hline \multicolumn{4}{|l|}{ Classificação IMC\# ${ }^{\#}$ n(\%) } \\
\hline Normal $^{\alpha}$ & $06(42,9)$ & $04(28,6)$ & 0,527 \\
\hline Sobrepeso $^{\alpha}$ & $02(14,3)$ & $04(28,6)$ & 0,414 \\
\hline Obesidade $^{\alpha}$ & $06(42,9)$ & $06(42,9)$ & 1,000 \\
\hline $\begin{array}{l}\text { Tempo de amputação } \\
\text { (meses) }^{\#}\end{array}$ & $\begin{array}{c}49(24- \\
216)\end{array}$ & $\begin{array}{c}30(18- \\
120)\end{array}$ & 0,295 \\
\hline \multicolumn{4}{|l|}{$\begin{array}{l}\text { Nível da amputação, n } \\
\text { (\%) }\end{array}$} \\
\hline $\operatorname{Transtibial}^{\alpha}$ & $02(14,3)$ & $05(35,7)$ & 0,257 \\
\hline Transfemural $^{\alpha}$ & $12(85,7)$ & $09(64,3)$ & 0,513 \\
\hline \multicolumn{4}{|l|}{$\begin{array}{l}\text { Altura da amputação, } \\
\text { n (\%) }\end{array}$} \\
\hline Proximal $^{\alpha}$ & $01(7,1)$ & - & - \\
\hline Medial $^{\alpha}$ & $08(57,1)$ & $10(71,4)$ & 0,637 \\
\hline Distal $^{\alpha}$ & $05(35,7)$ & $04(28,6)$ & 0,739 \\
\hline \multicolumn{4}{|l|}{ Protetização, n (\%) } \\
\hline Protetizado $^{\alpha}$ & $14(100,0)$ & $11(78,6)$ & 0,549 \\
\hline Não protetizado ${ }^{\alpha}$ & - & $03(21,4)$ & - \\
\hline $\begin{array}{l}\text { Presença de comor- } \\
\text { bidades, n (\%) }\end{array}$ & $03(21,4)$ & $12(85,7)$ & 0,020 \\
\hline $\mathrm{HAS}^{\alpha}$ & $02(14,3)$ & $03(21,4)$ & 0,655 \\
\hline Diabetes $^{\alpha}$ & - & $06(42,9)$ & - \\
\hline HAS e diabetes ${ }^{\alpha}$ & $01(7,1)$ & $03(21,4)$ & 0,317 \\
\hline
\end{tabular}

(Conclusão)

\begin{tabular}{|c|c|c|c|}
\hline \multirow[b]{2}{*}{ Variáveis } & \multicolumn{3}{|c|}{ AMPUTADOS $(n=28)$} \\
\hline & $\begin{array}{c}\text { GT } \\
(n=14)\end{array}$ & $\begin{array}{c}\text { GNT } \\
(n=14)\end{array}$ & $p$ \\
\hline \multicolumn{4}{|l|}{ DASI } \\
\hline Escore total ${ }^{\#}$ & $\begin{array}{c}23,2(2,7- \\
36,7)\end{array}$ & $\begin{array}{c}11,3(2,7- \\
31,4)\end{array}$ & 0,009 \\
\hline $\begin{array}{l}\mathrm{VO}_{2} \text { estimado (mL.kg }{ }^{-1} \text {. } \\
\left.\mathrm{min}^{-1}\right)^{\sigma}\end{array}$ & $19,6 \pm 4,4$ & $14,9 \pm 3,6$ & 0,006 \\
\hline \multicolumn{4}{|l|}{ Classificação do $\mathrm{VO}_{2}$} \\
\hline Muito fraca, $\mathrm{n}(\%)^{\alpha}$ & $14(100,0)$ & $14(100,0)$ & 1,000 \\
\hline
\end{tabular}

*Dados expressos em frequência, mediana, média \pm desvio-padrão e intervalo interquartil; ${ }^{\circ}$ Qui-quadrado; ${ }^{\varpi}$ teste $t$ de Student independente; ${ }^{*}$ Mann-Whitney $U$; \%: porcentagem; *IMC: Índice de Massa Corporal, segundo Deé; Lelovics, 2012:15 HAS: Hipertensão Arterial Sistêmica; DASI: Duke Activity Status Index for Cardiovascular Diseases; $\mathrm{VO}_{2}$ : consumo de oxigênio; $\mathrm{mL}$ : mililitros; $\mathrm{kg}$ : quilogramas; min: minutos; ${ }^{\#}$ Classificação IMC, segundo OMS, 2000. ${ }^{16}$

Também realizamos as análises com os indivíduos estratificados conforme o nível de amputação em grupo transtibial (GTT) e grupo transfemural (GTF). Apesar de não encontrar diferença significativa para o $\mathrm{VO}_{2}$ estimado entre os grupos [GTT 16,7 $\pm 4,1$ vs GTF $17,4 \pm 4,8$ (mL.kg-1.min-1), p=0,704], nós encontramos que GTF é composto por um maior número de indivíduos do sexo masculino [GTT, $\mathrm{n}=05$ (71\%) vs GTF, $\mathrm{n}=17(81 \%)$, $\mathrm{p}=0,011)$ e causa de amputação predominantemente traumática [GTT, $\mathrm{n}=02(29 \%)$ vs GTF, $\mathrm{n}=09(43 \%), \mathrm{p}=$ 0,008 ] quando comparado a GTT.

\section{DISCUSSÃO}

Dentre os principais achados deste estudo destacam-se um $\mathrm{VO}_{2}$ estimado muito fraco e associações entre o $\mathrm{VO}_{2}$ estimado e a idade dos indivíduos amputados no grupo avaliado como um todo e no grupo de amputados transfemurais. Sabe-se que com o envelhecimento há a redução significativa na capacidade aeróbia, o que reflete em menor capacidade do corpo em transportar oxigênio para os tecidos e pode ser considerado um fator de risco independente para mortalidade cardiovascular ${ }^{17,18,19}$.

No presente estudo também foi observada a presença de comorbidades em 53,6\% dos amputados, 
com maior frequência no GNT $(85,7 \%)$ quando comparado ao GT $(21,4 \%)$, e maior frequência de DM. Evidência demonstra que o DM promove a redução do $\mathrm{VO}_{2}$, além de resultar em diminuição da capacidade cardiorrespiratória e da tolerância ao exercício ${ }^{18,20}$. Isso ocorre pela insuficiência e/ou utilização incorreta da insulina, o que pode acarretar em uma série de distúrbios relacionados à síntese da glicose ingerida dos alimentos, prejudicando sua utilização como energia pelo nosso organismo ${ }^{19,21}$. Cabe salientar, que para promoção da qualidade do $\mathrm{VO}_{2}$ vem sendo estudado experimentalmente o uso de próteses ${ }^{22}$.

Outro fator relevante e que pode ter contribuído para um $\mathrm{VO}_{2}$ estimado fraco foi o excesso de peso encontrado nos indivíduos deste estudo. $\mathrm{O}$ excesso de peso pode provocar a uma diminuição da capacidade de trabalho físico e da eficiência cardíaca, bem como do $\mathrm{VO}_{2}^{23}$. Em adultos jovens, aqueles com valores de $\mathrm{VO}_{2}$ baixo apresentam maiores valores de IMC bem como altos valores de pressão arterial sistólica, classificando-os com elevado risco cardiovascular, quando comparados aos que apresentaram altos valores de $\mathrm{VO}_{2}$ e baixos valores de $\mathrm{IMC}^{24}$.

Ao estratificar os indivíduos amputados, por motivo de amputação, encontramos maior $\mathrm{VO}_{2}$ estimado e melhor capacidade funcional (Tabela 2) no GT em comparação com o GNT. Amputações por causas vasculares provocam maior gasto energético quando comparadas às causas traumáticas, pelas doenças de base ${ }^{25-28}$. Isto faz sentido, haja vista que $76 \%$ do GNT apresentavam diabetes mellitus como doença de base coexistindo com as consequências da amputação, em comparação a um único sujeito amputado (7\%) do GT. Ressaltamos ainda que os amputados do GT eram significativamente mais jovens que GNT, contribuindo para melhor capacidade funcional.

Alguns fatores limitantes do presente estudo devem ser destacados como o fato dos amputados aqui estudados serem beneficiários do Sistema Único de Saúde (SUS), o qual dispensa próteses tecnologicamente menos favorecidas, bem como não dispensa liner o que dificulta o encaixe da prótese, quando comparadas a serviços não públicos. Desta forma, as próteses utilizadas pelos indivíduos deste estudo não permitem a esses amputados algumas atividades, do tipo correr ou movimentar-se muito, fator este que implica nessa redução do nível de atividade física desse grupo. Cabe ressaltar que este estudo também apresenta como limitações o número amostral, bem como a realização por método de conveniência.

Portanto, destaca-se como implicação clínica do estudo a importância de elaboração de próteses que favoreçam o gasto energético de amputados, proporcionando, desta forma, aumento da capacidade funcional e desempenho nas AVDs. Adicionalmente, o estudo contribui para o reconhecimento de que estratégias de prevenção da amputação bem como o tratamento precoce para os casos não traumáticos são de extrema relevância para o crescimento de sobrevida.

\section{CONCLUSÃO}

Diante do exposto, é possível inferir que os amputados de membro inferior unilateral apresentaram diminuída capacidade funcional e $\mathrm{VO}_{2}$ estimado muito fraco. A idade, a obesidade, o nível transfemural e a causa não traumática da amputação influenciaram diretamente o baixo $\mathrm{VO}_{2}$ estimado.

\section{REFERÊNCIAS}

1. Marques AMFB, Vargas MAO, Schoelle SD, Kinoshita EY, Ramos FRS, Trombetta AP. O cuidado à saúde à pessoa com amputação: análise na perspectiva da bioética. Texto Contexto Enferm. 2014;23(4): 898906.

2. Humphries MD, Brunson A, Hedayati N, Romano $\mathrm{P}$, Melnkow JAL. Amputation risk in patients with diabetes mellitus and peripheral artery disease using Statewide data. Ann Vasc Surg. 2016;30: 1-9.

3. Knežević A, Salamon T, Milankov M, Ninković S, Jeremić Knežević M, TomaševićTodorović S. Assessmentofqualityoflife in patientsafterlowerlimbamputation. MedPregl. 2015;68(3): 103-108.

4. OmokeNI, Chukwu CO, Madubueze CC, Egwu AN. Traumatic extremity amputation in a Nigerian setting: patterns and challenges of care. IntOrthop. 2012;36(3): 613-618.

5. Garcia MMN, Lima JRP, Costa Junior JD, Freire HAOL, Mazilão JP, Vicente EJD. Energy expenditureand cardiovascular response to traumatic lower limb amputees' gait. Fisioter. Mov. 2015;28(2): 259-268. 
6. Bona RL, Tartaruga LAP. Comparação do consumo energético e de aspectos mecânicos da caminhada de amputados transfemurais que utilizam prótese com microprocessador ou convencional: uma revisão. Pensar a prática. 2011;14(1): 1-14.

7. Starholm IM, Mirtaheri P, Kapetanovic N, Versto T, Skyttemyr G, Westby FT et al. Energy expenditure of transfemoral amputees during floor and treadmill walking with different speeds. POI. 2016;40(3): 336342 .

8. Stauffer S. Estimated Maximal Oxygen Consumption of Transfemoral Amputees Utilizing the Ebbeling Treadmill Test. Senior Honors Theses. 2016; 1-28.

9. Tang WHW, Topol EJ, Fan Y, Wu Y, Cho L, Steveson C et al. Prognostic valeu of estimated functional capacity incremental to cardiac biomarkers in stable cardiac patients. J Am Heart Assoc. 2014;3(5):000960.

10. Grodin JL, Hammadah M, Fan Y, Hanzen SL, Tang WH. Prognostic Value of Estimating Functional Capacity Usingthe Duke Activity Status Index in Stable Patients with Chronic Heart Failure. J CardFail. 2015;21(1): 44-50.

11. Wijeysundera DN, Pearse RM, Shulman MA, Abbott TEF, Torres E, Croal BL et al. Measurement of Exercise Tolerance before Surgery (METS) study: a protocol for aninternationalmulticentreprospectivecohortstudyofcardiopulmonaryexercisetesting prior to major non-cardiacsurgery. BMJ Open. 2016;6(3): 010359.

12. Coutinho-Myrrha MA, Dias RC, Fernandes AA, Araújo CG, Hlatky MA, Pereira DG et al. Duke Activity Status Index em doenças cardiovasculares: validação de tradução em português. Arquivos Brasileiros de Cardiologia. 2014;102(4): 383-90.

13. TavaresLA, Barreto Neto J, Jardim JR, Souza GMC, Hlatky MA, Nascimento OA. Adaptação cultural e avaliação da reprodutibilidade do Duke Activity Status Index para pacientes com DPOC no Brasil. J.braspneumol. 2012;38(6): 684-92.

14. Cooper KH. The aerobicsway. Bantam Books 1982.

15. Deé K, Lelovics Z. Correct determination of body mass index in people with lower limb amputation. Advanced Research in Scientific Areas. 2012; 7(3): 2133-37.

16. World Health Organization - WHO. Obesity: preventing and managing the global epidemic. Report of a World Health Organization Consultation. Geneva:
World Health Organization. WHO. 2000. p. 256.

17. Carvalho Filho ET. Fisiologia do envelhecimento. In: Tratado de Gerontologia, 2. ed., Atheneu. 2007.

18. Green S, Egaña M, Baldi JC, Lamberts R, Regensteiner JG. Cardiovascular control during exercise in type 2 diabetes mellitus. Journal of Diabetes Research. $2015 ; 2015$.

19. Maughan R, Gleeson M, Greenhaff PL. Bioquímica do exercício e do treinamento. Manole. 2000.

20. Parasoglou P, Rao S, Slade JM. Declining skeletal muscle function in diabetic peripheral neuropathy. ClinTher. 2017;39(6): 1085-1103.

21. Bona RL, Aldabe D, Ribeiro JL. Avaliação do gasto energético em pacientes amputados de membro inferior protetizados. Arq Sanny Pesq Saúde. 2008;1(2): 98-108.

22. Gardinier ES, Kelly BM, Wensman J, Gates DH. A controlled clinicaltrial of a clinically-tuned power edankle prosthesis in people with transtibial amputation. Clinical rehabilitation. 2018;32(3): 319-29.

23. Jong AT, Gallagher MJ, Sandberg KR, Lillystone MA, Spring T, Franklin BA et al. Peak oxygen consumption and the minute ventilation/carbono dioxide production relations lope in morbidly obese men and women: influence of subject effort and body mass index. ASPC. 2008;11(2): 100-105.

24. Fernandes RA, Codogno JS, Campos EZ, Rodrigues EQ, Sousa S, Balikian Júnior P et al. Consumo máximo de oxigênio e fatores de risco cardiovascular em adultos jovens. RBAFS. 2009;14(2): 96-103.

25. Czerniecki JM. Rehabilitation in limbd eficiency. 1. Gait and motion analysis. Arch Phys Med Rehabil. 1996; 77(3): S3-S8.

26. Pinzur MS. Gaitanalysis in peripheral vascular insufficiency through-knee amputation. J Rehabil Res Dev. 1993;30(4): 388-92.

27. Waters RL, Perry J, Antonelli D, Hislop H. Energy cost of walking of amputees: the influence of level of amputation. J Bone Joint Surg Am.1976; 58(1): 42-6.

28. Waters RL, Mukroy S. The energy expenditure of normal and patuologic gait. Gait Posture. 1999;9(3): 207-31. 
Recebido em: 03/07/2018

Aceito em: 08/08/2018 